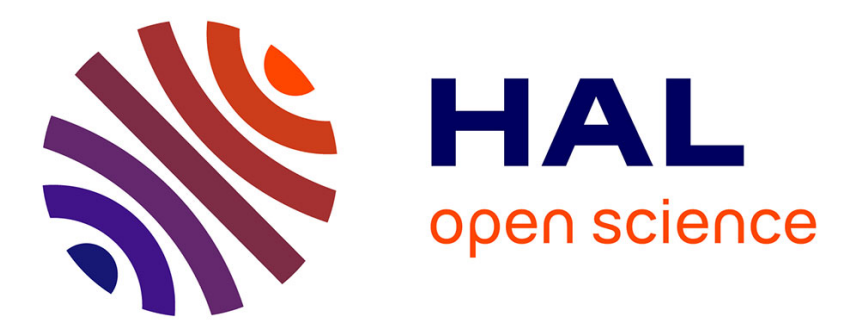

\title{
Beyond the Magnetic Domain Matching in Magnetic Exchange Coupling
}

Hélène Magnan, Odile Bezencenet, Dana Stanescu, R Belkhou, A Barbier

\section{To cite this version:}

Hélène Magnan, Odile Bezencenet, Dana Stanescu, R Belkhou, A Barbier. Beyond the Magnetic Domain Matching in Magnetic Exchange Coupling. Physical Review Letters, 2010, 105, pp.097204. 10.1103/PhysRevLett.105.097204 . cea-01481361

\section{HAL Id: cea-01481361 https://hal-cea.archives-ouvertes.fr/cea-01481361}

Submitted on 2 Mar 2017

HAL is a multi-disciplinary open access archive for the deposit and dissemination of scientific research documents, whether they are published or not. The documents may come from teaching and research institutions in France or abroad, or from public or private research centers.
L'archive ouverte pluridisciplinaire HAL, est destinée au dépôt et à la diffusion de documents scientifiques de niveau recherche, publiés ou non, émanant des établissements d'enseignement et de recherche français ou étrangers, des laboratoires publics ou privés. 


\title{
Beyond the Magnetic Domain Matching in Magnetic Exchange Coupling
}

\author{
H. Magnan, ${ }^{1}$ O. Bezencenet, ${ }^{1}$ D. Stanescu, ${ }^{1}$ R. Belkhou, ${ }^{2}$ and A. Barbier ${ }^{1, *}$ \\ ${ }^{1}$ CEA Saclay, DSM/IRAMIS/SPCSI, F-91191 Gif-sur-Yvette Cedex, France \\ ${ }^{2}$ Synchrotron SOLEIL, L'Orme des Merisiers, Saint-Aubin, F-91192 Gif-sur-Yvette Cedex, France
}

(Received 22 March 2010; published 26 August 2010)

\begin{abstract}
We report a new macroscopic first-field-induced magnetic anisotropy for $\mathrm{Co} / \alpha-\mathrm{Fe}_{2} \mathrm{O}_{3}(0001)$ layers, a prototypical ferromagnetic-antiferromagnetic interface for which the antiferromagnetic film has small inplane magnetic anisotropy as compared to the interface coupling. We demonstrate that the effect is due to a first-field-induced irreversible magnetic domain motion in the antiferromagnetic layer, dragged by the ferromagnetic Co one. Whereas the initial domain matching is lost, the macroscopic manifestations of the exchange coupling remain stable. Therefore, the initial domain matching probably has only a marginal role in the explanation of the magnetic exchange coupling.
\end{abstract}

DOI: 10.1103/PhysRevLett.105.097204

PACS numbers: 75.30.Gw, 75.30.Et, 75.70.Kw

The magnetic exchange coupling phenomenon, discovered in 1956 by Meiklejohn and Bean [1], occurs at ferromagnetic- (FM-)antiferromagnetic (AFM) interfaces and has found a broad range of practical applications with high industrial impact. It is extensively used to set a reference magnetization direction in magnetic sensors and spintronics devices [2]. It is commonly accepted that the unidirectional magnetic exchange anisotropy can be induced (i) by growing a FM layer under an external magnetic field on an AFM having high in-plane magnetic anisotropy or (ii) by cooling down a FM-AFM bilayer from above the Néel temperature $\left(T_{N}\right)$ of the AFM layer (and below the Curie temperature $\left(T_{C}\right)$ of the ferromagnetic film) in a saturating external magnetic field (field cooling process). The magnetic exchange coupling manifests itself, up to the blocking temperature, through a combination of (i) an exchange bias field $H_{E}$ (corresponding to a hysteresis loop shift along the field axis) and/or (ii) an enhancement of the coercive field $H_{C}[3,4]$. Recent studies evidenced the importance of the AFM domain structure near the interface since FM spins were found to align along the AFM spin direction as, e.g., in $\mathrm{Co} / \mathrm{LaFeO}_{3}(001)$ [5], $\mathrm{Co} / \mathrm{NiO}(001)$ [6], MnIr [7], and $\mathrm{Co} / \alpha-\mathrm{Fe}_{2} \mathrm{O}_{3}$ [8] bilayers. The direct correlation between the FM and AFM domain structures mediated through interfacial uncompensated AFM spins has been proposed as being the key feature of the FM-AFM magnetic exchange coupling. In the present study, we use an uncommon approach to induce a magnetic anisotropy. We found that the direction of the first applied magnetic field induces a uniaxial magnetic anisotropy for layers deposited in a zero magnetic field and without field cooling. More importantly, we show that the first field also breaks the initial magnetic domain matching without canceling the magnetic exchange coupling.

As a matter of fact, additional noteworthy effects have been neglected as, e.g., the "training effect" characterized by the progressive decrease of exchange bias and coercivity upon repeated cycling of the hysteresis loop [9-11].
This effect suggests that the AFM spin structure, which is responsible for the loop pinning and coercivity change, may not be static upon cycling. It has also been shown that a rotating external field could change the AFM spin structure by dragging the spins away from their initial field cooled orientation $[12,13]$; this phenomenon is more pronounced when the uniaxial magnetic anisotropy of the AFM layer is small [13]. Different models have been proposed to explain magnetic exchange bias for both compensated and uncompensated interfaces [9]. From these models it appears that, if the anisotropy of a AFM film is too low, no exchange bias is expected and consequently investigations of such systems are scarce.

Hematite $\left(\alpha-\mathrm{Fe}_{2} \mathrm{O}_{3}\right)$ is the most stable antiferromagnetic iron oxide with high Néel temperature $(955 \mathrm{~K})$. At room temperature its AFM spin moments lie in the basal (0001) plane; neighboring planes are coupled antiferromagnetically in a threefold magnetic domain structure due to equivalent spin orientations differing by $120^{\circ}[14,15]$ with a small in-basal-plane magnetic anisotropy [16]. Co is a ferromagnetic $3 d$ transition metal with a high Curie temperature $(1388 \mathrm{~K})$. The Co-hematite interface may thus be considered as a model system for the study of AFM-FM coupling with a low AFM in-plane anisotropy. However, only a small value of $H_{E}$ is expected.

Our $\mathrm{Co} /(20 \mathrm{~nm}) \alpha-\mathrm{Fe}_{2} \mathrm{O}_{3}(0001)$ films were deposited by atomic oxygen assisted molecular beam epitaxy on $\alpha-\mathrm{Al}_{2} \mathrm{O}_{3}(0001)$ [17] or $\mathrm{Pt}(111)$ [18]. They were regenerated after air exposure as described in Ref. [18]. Cobalt was deposited at room temperature from a high purity rod heated by electron bombardment in a zero magnetic field. In our previous structural investigations we have shown that room temperature grown Co layers adopt a nucleation-growth-coalescence scheme with a critical thickness of about $2-3 \mathrm{~nm}$ along with a poor crystalline quality that includes all possible twin structures [19]. The interface interdiffusion is limited to the very first plane [20] leading to metallic (uncompensated) interfacial $\mathrm{Fe}$ and oxidized 
Co. $H_{C}$ varies linearly with $1 / t_{\mathrm{Co}}$ (where $t_{\mathrm{Co}}$ is the Co thickness) demonstrating the interfacial nature of the coupling [21]. The exchange field $H_{E}$ is found to be negligible, which is not uncommon for ferromagnetic films deposited on hematite [22].

The films were investigated using two UHV approaches: macroscopic [surface magneto-optical Kerr effect (SMOKE)] and submicroscopic [dichroic x-ray photoemitted electron microscopy (XPEEM)]. For ex situ measurements, such as magnetic characterization using a vibrating sample magnetometer, the samples were protected against oxidation by a $\sim 2 \mathrm{~nm}$ thick $\mathrm{Au}$ capping layer. All the measurements were carried out at room temperature. The XPEEM measurements were carried out at the ELETTRA and SLS synchrotrons (Nanospectroscopy and SIM beam lines, respectively) using an ELMITEC GmBH LEEM V instrument. For the SMOKE measurements [23] the magnetic field was applied in the surface plane of the sample and in the incidence plane. By convention $\alpha_{H}$ is the azimuthal angle between the in-surface-plane [1120] direction of hematite and the magnetic field. The angle of incidence of the laser beam was $45^{\circ}$, and we used $s$ and $p$ incident polarization for longitudinal (LMOKE) and transversal (TMOKE) measurements, respectively.

The Co-hematite magnetic exchange coupling leads to a large increase of the coercive field, $H_{C}=5$ Oe for $0.3 \mathrm{~nm}$ $\mathrm{Co} / \alpha-\mathrm{Al}_{2} \mathrm{O}_{3}$ (without AFM layer) and $H_{C}=137$ Oe for a sample with the same Co thickness evaporated on a hematite layer [8]. This $H_{C}$ enhancement is likely due to the large interfacial coupling energy as compared to the low in-plane anisotropy of the AFM layer [16]. When the FM layer rotates, it drags the AFM spins irreversibly, which increases the FM coercivity [9].
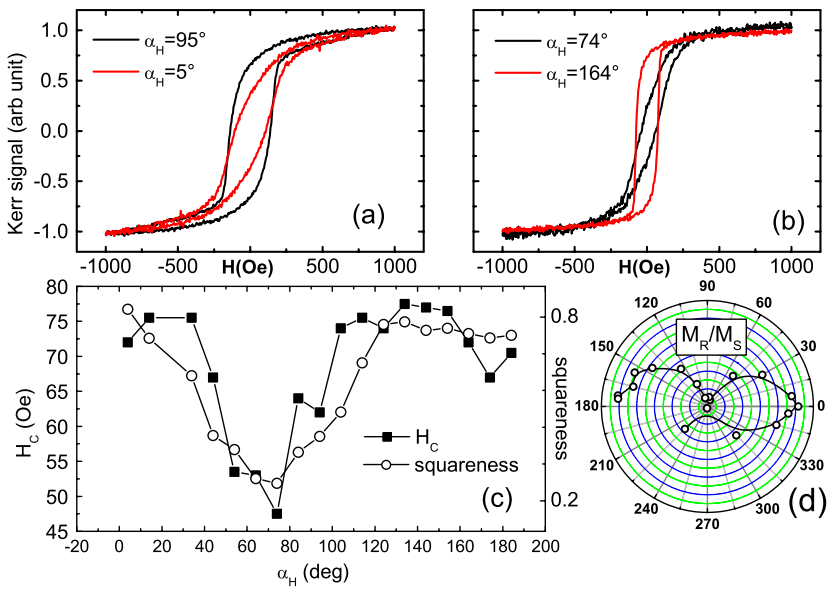

FIG. 1 (color online). Longitudinal Kerr hysteresis loop at room temperature for Co films evaporated on hematite layer (a) sample 1, $1 \mathrm{~nm}$ of Co, (b) sample 2, $2 \mathrm{~nm}$ of Co. The corresponding $\alpha_{H}$ angles are indicated. (c) $H_{C}$ and squareness $\left(M_{R} / M_{S}\right)$ of sample 2 as function of $\alpha_{H}$. (d) Polar representation of $M_{R} / M_{S}$. Sample similar to (c) with same composition. The radial scale extends from 0.3 to 0.9 .
Let us now examine the macroscopic magnetic coupling with respect to the initial field direction. Figures 1(a) and 1(b) show longitudinal hysteresis loops recorded at different $\alpha_{H}$ for two different thicknesses of cobalt. The loop shape differences for the two orthogonal directions clearly indicate an in-plane magnetic anisotropy for these films. Interestingly, the plot of the coercive field and squareness $\left(M_{R} / M_{S}\right)$ as a function of $\alpha_{H}$ [Figs. 1(c) and 1(d)] evidences a uniaxial anisotropy (twofold symmetry), i.e., simultaneous minima of $H_{C}$ and $M_{R} / M_{S}$. The comparison of Figs. 1(a) and 1(b) shows that the easy magnetic axis does not lie along a particular crystallographic direction. In order to rule out a possible substrate miscut effect, we cut a $\mathrm{Fe}_{2} \mathrm{O}_{3} / \mathrm{Al}_{2} \mathrm{O}_{3}(0001)$ sample in two parts and studied them in two different configurations. They were mounted in two different azimuthal direction on the sample holder in the Kerr chamber prior to Co deposition. After deposition of cobalt, both samples exhibit in-plane anisotropy, but the easy axis was not found in the same crystallographic direction. As we can see in Fig. 2(a), the easy axis is always aligned with the direction of the "first field," i.e., the direction of the first hysteresis loop just after the evaporation. Moreover, no in-plane anisotropy is observed when cobalt is evaporated directly on alumina; thus, we can conclude that the anisotropy is clearly related to the presence of the hematite layer. The usual anisotropy sources like off-normal deposition or vicinal substrates can safely be excluded here.

We have evaluated the stability of this in-plane anisotropy. The anisotropy remained unaffected after an additional $\mathrm{Co}$ or $\mathrm{Au}$ deposition. The anisotropy was also unchanged after several months of air storage. A demagnetizing procedure $\left(M_{S} \sim 0\right)$ as well as the exposure to a large magnetic field $\left(1.8 \mathrm{~T} \gg 4 \pi M_{S}\right)$ proved to be unable to modify the initial easy axis direction. Obviously, the mechanism of the establishment of this anisotropy occurs during
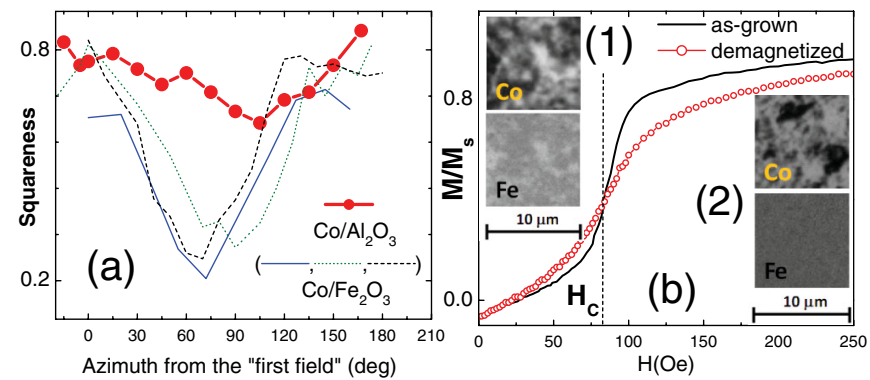

FIG. 2 (color online). (a) Squareness of hysteresis loop recorded on $2 \mathrm{~nm}$ Co on hematite films as function of the azimuth from the first field: $\alpha_{H}=95^{\circ}$ (straight line), $\alpha_{H}=$ $5^{\circ}$ (dotted line), and $\alpha_{H}=4^{\circ}$ (dashed line); Co evaporated on alumina (circles). (b) Virgin magnetic curves obtained on $2 \mathrm{~nm} \mathrm{Au} / 1.4 \mathrm{~nm} \mathrm{Co} / \mathrm{Fe}_{2} \mathrm{O}_{3}$ for the as-grown sample and after demagnetization. FM and AFM XPEEM domain structures for $1.6 \mathrm{~nm} \mathrm{Co} / \mathrm{Fe}_{2} \mathrm{O}_{3}(20 \mathrm{~nm}) / \mathrm{Pt}(111)$ in (1) virgin state and (2) after demagnetization. 
the first magnetization. In order to confirm this assumption, we studied the "virgin curve" (i.e., the first magnetization curve) of an as-deposited $2 \mathrm{~nm} \mathrm{Au} / 1.4 \mathrm{~nm} \mathrm{Co} / \mathrm{Fe}_{2} \mathrm{O}_{3}$ sample [Fig. 2(b)]. We observe a low initial susceptibility, showing that the domain walls are pinned for low magnetic fields. In Fig. 2(b) we have also plotted the virgin curve obtained after a full demagnetization procedure of the sample. It is completely different from the first virgin curve; the initial magnetic susceptibility is now significantly larger. This shows that after magnetization the sample is irreversibly modified.

To get insights in the microscopic interface magnetic domain structure we have studied $\mathrm{Co} / \mathrm{Fe}_{2} \mathrm{O}_{3} / \mathrm{Pt}(111)$ bilayers by XPEEM after exposure to different magnetic fields, taking advantage of the dichroic effects on the $\mathrm{L}_{2,3}$ edges of $\mathrm{Co}$ and $\mathrm{Fe}$ as described in Ref. [5]. The $\mathrm{Co}$ and $\mathrm{Fe}$ XMCD (circular dichroism) XPEEM images allow us to investigate the FM domains, respectively, within the Co layers and the Fe interface. Meanwhile, the Fe XMLD (linear dichroism) XPEEM images evidence the AFM domain structure in the hematite substrate. In the virgin state [Figs. 3(a)-3(c)] the Co ferromagnetic spins are aligned, domain by domain, with the spin direction in the interfacial uncompensated metallic Fe spins and the underlying AFM spins. When a small (compared to $H_{C} \sim 45 \mathrm{Oe}$ ) magnetic field is applied, the magnetic domains of Co are modified as expected for a ferromagnet [Fig. 3(d)], larger domains align with the external field and they drag the magnetic domains of the uncompensated layer [Fig. 3(e)]. The do-

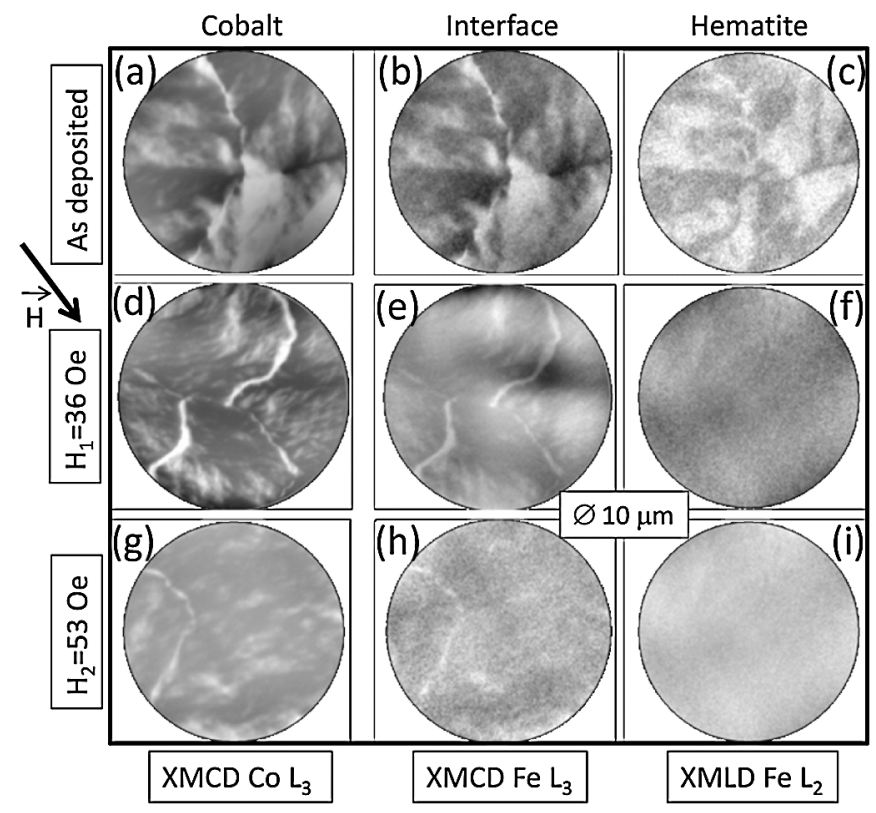

FIG. 3. Dichroic XPEEM images recorded at the $\mathrm{Co} \mathrm{L}_{3}$ (a),(d), (g), Fe $\mathrm{L}_{3}$ (b),(e),(h), and $\mathrm{Fe} \mathrm{L}_{2 A, B}$ (c),(f),(i) edges for a $2.5 \mathrm{~nm} \mathrm{Au} / 3.5 \mathrm{~nm} \mathrm{Co} / 20 \mathrm{~nm} \mathrm{Fe}{ }_{2} \mathrm{O}_{3} / \mathrm{Pt}(111)$ sample. Virgin state (a)-(c) and effect of increasing magnetic fields. The regions are different since the sample had to be moved to apply the magnetic field. mains of the AFM layer [Fig. 3(f)] are also dragged and the apparent domain structure vanishes. This can be due to (i) AFM domains larger than the XPEEM field of view, (ii) AFM domains breaking up in domains smaller than the XPEEM spatial resolution. or (iii) an alignment of the AFM spin with the external magnetic field (via the FM domain motion and the dragging effect) leading to a uniaxial state and thus to an homogeneous XMLD response with domain wall widths below the XPEEM resolution. The two first assumptions can be excluded because the investigation of many sample regions did not reveal the presence of domain walls and a vanishing domain size would not allow us to keep large $H_{C}$ values. This behavior is confirmed for field values above $H_{C}$ [Figs. 3(g)-3(i)] where the FM and AFM layers almost no longer show a domain configuration. At the $H$ value corresponding to the divergence of the virgin curve $\left(H \sim H_{C}\right)$ the AFM domain structure becomes independent of the ferromagnetic domain structure. Importantly, demagnetizing the sample restores observable Co domains but leaves the antiferromagnet in the uniaxial domain state; i.e., the magnetic domain matching is not restored (Fig. 2).

LMOKE and TMOKE allow us to establish a complete understanding of the magnetic anisotropy. A $10 \mathrm{~nm}$ thick Co layer was deposited on a $10 \mathrm{~nm}$ thick $\mathrm{Fe}_{2} \mathrm{O}_{3} / \mathrm{Al}_{2} \mathrm{O}_{3}$ layer previously annealed in air at $1100 \mathrm{~K}$ for $2 \mathrm{~h}$ to improve the crystalline structure. This sample was capped with $3.5 \mathrm{~nm} \AA$ of Au. Experimental measurements are shown in Fig. 4 for different azimuthal angles (by convention $0^{\circ}$ is the direction of the first field). The coercive field is about 2 times larger than for the nonannealed $20 \mathrm{~nm}$ hematite layer (160 Oe instead of 75 Oe) [see Fig. 1(b)]. The shape of the hysteresis loops is typical for uniaxial

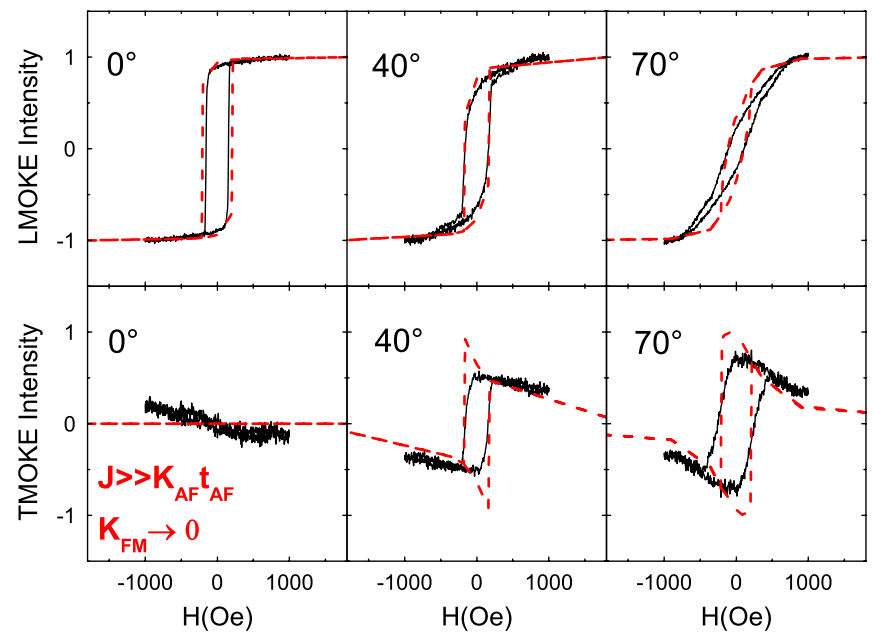

FIG. 4 (color online). LMOKE (top) and TMOKE (bottom) measurement performed on $10 \mathrm{ML} \mathrm{Co} / 10 \mathrm{~nm}$ annealed $\mathrm{Fe}_{2} \mathrm{O}_{3}$ (see text) for different azimuthal angles with respect to the firstfield direction. Straight line, experiment; dotted line, model with $t_{\mathrm{FM}}=2 \mathrm{~nm}, t_{\mathrm{AFM}}=10 \mathrm{~nm}, M_{\mathrm{FM}}=1400 \mathrm{emu} / \mathrm{cm}^{3}$, and $K_{\mathrm{AFM}}=2.5 \times 10^{5} \mathrm{erg} / \mathrm{cm}^{3}=2.5 \times 10^{4} \mathrm{~J} / \mathrm{m}^{3}$ (see text). 
anisotropy. We used the Meiklejohn model [24], which assumes a coherent rotation of the magnetization to simulate the hysteresis loops. The energy per unit area can be written [9] as

$$
\begin{aligned}
E= & -H M_{\mathrm{FM}} t_{\mathrm{FM}} \cos (\theta-\beta)+K_{\mathrm{FM}} t_{\mathrm{FM}} \sin ^{2}(\beta) \\
& +K_{\mathrm{AFM}} t_{\mathrm{AFM}} \sin ^{2}(\alpha)-J \cos (\beta-\alpha),
\end{aligned}
$$

where $H, M_{\mathrm{FM}}, t_{\mathrm{FM}}, t_{\mathrm{AFM}}, K_{\mathrm{FM}}, K_{\mathrm{AFM}}$, and $J$ are, respectively, the applied field, the saturation magnetization, the FM thickness, the AFM thickness, the FM anisotropy, the $\mathrm{AFM}$ anisotropy, and the interface coupling constant. $\beta, \alpha$, and $\theta$ are, respectively, the angles between magnetization and the FM anisotropy axis, the AFM spin direction and the AFM anisotropy axis, and the applied field and the FM anisotropy axis. The terms describe, respectively, the effects of the field on the FM layer, of the FM anisotropy, of the AFM anisotropy, and of the interface coupling. Our interface allows further approximations: (i) $K_{\mathrm{FM}} \rightarrow 0$ since Co adopts many in-plane variants [19], (ii) $J \gg K_{\mathrm{AFM}} t_{\mathrm{AFM}}$ since the in-plane anisotropy of sixfold hematite is small. The second assumption implies $\beta \approx \alpha$, i.e., the AFM and the FM spin rotate together, the term including $J$ will vanish upon derivation, and the exchange bias field $H_{E}$ will be zero. Finally, the energy equation becomes identical here to the Stoner-Wohlfahrt model [25] which takes into account neither temperature effects nor domain wall activation that may lead to additional effects [26]. Minimizing the equation with respect to $\beta$, one can calculate the coercive field when the field is applied along the easy axis $(\theta=0): H_{C}=\left(2 K_{\mathrm{AFM}} t_{\mathrm{AFM}}\right) /\left(M_{\mathrm{FM}} t_{\mathrm{FM}}\right)$.

$H_{C}$ varies as $1 / t_{\mathrm{FM}}$ as found experimentally [8]. The best loop simulation (Fig. 4) is obtained for $K_{\mathrm{AFM}}=$ $2.5 \times 10^{5} \mathrm{erg} / \mathrm{cm}^{3}$, which is much larger than the sixfold in-plane anisotropy constant measured on bulk hematite [16] $\left(0.56 \mathrm{erg} / \mathrm{cm}^{3}\right)$. Our $K_{\mathrm{AFM}}$ is of the order of magnitude of the anisotropy measured on hematite nanoparticles [27] and/or the one of AFM oxides like CoO. The $\sim 0.4 \mathrm{~nm}$ thick interface intermixing is known [20] and may, to some extent, strengthen $K_{\mathrm{AFM}}$. Our first field induced in-plane anisotropy is thus likely linked to reduced dimensions and/ or interface composition effects.

In summary, we have demonstrated that $\mathrm{Co} / \mathrm{Fe}_{2} \mathrm{O}_{3}$ films have an in-plane magnetic anisotropy induced by the first field that irreversibly modifies the sample magnetic structure leading to an easy axis of the magnetic anisotropy. We have shown that the hysteresis loop can be simulated by a simple model assuming coherent rotation and an in-plane magnetic anisotropy in the AFM layer induced by the first field. Moreover, the magnetic domain matching corresponds only to the initial virgin state and its loss does not affect the overall magnetic exchange coupling parameters such as the $H_{C}$ value. The present findings show that the importance of the magnetic domain matching has probably been overestimated in the understanding of the exchange coupling phenomenon. As a matter of fact, domain matching does not imply the presence of a significant $H_{E}$ value, and the loss of the initial domain matching does not imply a loss of magnetic exchange coupling. Our results also open a promising route to tailor the magnetic behavior of spintronic sensors using low anisotropy AFM films by taking advantage of the magnetic domain dragging effect that we have evidenced here.

The beam line staffs are acknowledged for efficient help during measurements. The research leading to these results has received funding from the European Community's Seventh Framework Programme (FP7/2007-2013).

*Corresponding author. abarbier@cea.fr

[1] W. H. Meiklejohn and C. P. Bean, Phys. Rev. 102, 1413 (1956).

[2] S. S. P. Parkin et al., Proc. IEEE 91, 661 (2003).

[3] M.D. Stiles and R.D. McMichael, Phys. Rev. B 63, 064405 (2001).

[4] C. Mocuta, A. Barbier, S. Lafaye, P. Bayle-Guillemaud, and M. Panabiere, Phys. Rev. B 68, 014416 (2003).

[5] F. Nolting et al., Nature (London) 405, 767 (2000).

[6] H. Ohldag et al., Phys. Rev. Lett. 86, 2878 (2001).

[7] T. Eimüller et al., Appl. Phys. Lett. 85, 2310 (2004).

[8] O. Bezencenet et al., J. Phys. Conf. Ser. 100, 072027 (2008).

[9] J. Nogues and I. K. Schuller, J. Magn. Magn. Mater. 192, 203 (1999).

[10] C. Binek, X. He, and S. Polisetty, Phys. Rev. B 72, 054408 (2005).

[11] M. Fecioru-Morariu, J. Wrona, C. Papusoi, and G. Guntherodt, Phys. Rev. B 77, 054441 (2008).

[12] S. Urazhdin and C. L. Chien, Phys. Rev. B 71, 220410 (2005).

[13] J. Olamit and K. Liu, J. Appl. Phys. 101, 09E508 (2007).

[14] C. G. Shull, W. A. Strauser, and E. O. Wollan, Phys. Rev. 83, 333 (1951).

[15] R. Nathans, S. J. Pickart, H. A. Alperin, and P. J. Brown, Phys. Rev. 136, A1641 (1964).

[16] P. J. Besser, A. H. Morrish, and C. W. Searle, Phys. Rev. 153, 632 (1967).

[17] S. Gota, M. Gautier-Soyer, and M. Sacchi, Phys. Rev. B 64, 224407 (2001).

[18] A. Barbier et al., Phys. Rev. B 72, 245423 (2005).

[19] O. Bezencenet et al., Phys. Rev. B 81, 085419 (2010).

[20] O. Bezencenet et al., Surf. Sci. 601, 4321 (2007).

[21] C. Leighton et al., Phys. Rev. B 65, 064403 (2002).

[22] J. Dho, C. W. Leung, Z. H. Barber, and M. G. Blamire, Phys. Rev. B 71, 180402 (2005).

[23] Z. Q. Qiu and S. D. Bader, J. Magn. Magn. Mater. 200, 664 (1999).

[24] W. H. Meiklejohn, J. Appl. Phys. 33, 1328 (1962).

[25] E. C. Stoner and E.P. Wohlfarth, Phil. Trans. R. Soc. A 240, 599 (1948).

[26] S. Rohart et al., Phys. Rev. Lett. 104, 137202 (2010).

[27] F. Bødker and S. Mørup, Europhys. Lett. 52, 217 (2000). 